\title{
Online-Vorlesungen mit dem Paella-Player
}

\author{
R. Stelzer \\ Professur Konstruktionstechnik/CAD, Institut für Maschinenelemente und Maschinenkonstruktion, TU Dresden
}

\begin{abstract}
Online-Vorlesungen können in verschiedenen Arten angeboten werden. Dazu gehören das Echtzeit-Streaming, vertonte Powerpoints sowie einfache Videos. Alle diese Angebote haben Vorund Nachteile. So ist bei großen Hörerzahlen das direkte Streaming häufig aus technischen Gründen unmöglich. Vertonte Powerpoints sind für den Studenten nur sehr unkomfortabel zu nutzen. Videos haben da eine Reihe von Vorteilen. Allerdings hat es sich gezeigt, dass es sehr sinnvoll ist, wenn gleichzeitig sowohl die Präsentation als auch der Dozent sichtbar sind. Dies ermöglicht der von der Universitat Politècnica de València entwickelte Paella-Player. Die Funktionalität sowie die Voraussetzungen der Nutzung dieses Players sollen im Beitrag vorgestellt werden.

Online lectures can be offered in different ways. These include real-time streaming, powerpoints set to music and simple videos. All of these offerings have advantages and disadvantages. For example, with large numbers of listeners, direct streaming is often impossible for technical reasons. Powerpoints with sound are very uncomfortable for students to use. Vi-deos have a number of advantages. However, it has been shown that it is very useful if both the presentation and the lecturer are visible at the same time. This is made possible by the Paella Player developed by the Universitat Politècnica de València. The functionality as well as the requirements for using this player will be presented in the article.
\end{abstract}

*Corresponding author: ralph.stelzer@tu-dresden.de 


\section{Intention}

Ich möchte über die Nutzung des Paella-Players für die online-Vorlesungen in Konstruktionslehre und Informatik berichten.

Nachdem klar war, dass die Vorlesungen in den großen Grundlagenvorlesungen im Sommersemester 2020 nur online umgesetzt werden können, hatte ich verschiedene Formate versucht.

Dabei wurde eine online-Vorlesung in Echtzeit wegen der großen Hörerschaft $(>500 \mathrm{Hö-}$ rer) nicht weiter in Betracht gezogen.

Der erste Versuch waren Vorlesungen als vertonte Powerpoints. Diese wurden allerdings in den mir übergebenen Bewertungen zu fast $100 \%$ abgewählt. Die größten Probleme bei dieser Variante sind:

- Freies Wählen bestimmter Passagen ist kaum möglich

- Wiederholung einzelner Phrasen ist schwierig

- Aussetzer im Audio-Stream beim Folienwechsel;

Dies liegt vor allem daran, dass man oft keine Pause beim Sprechen macht, wenn die Folien weiterschalten. Da Powerpoint, den Ton jedoch direkt in den Folien platziert, kommt es notgedrungen zu diesen Aussetzern.

Im zweiten Versuch habe ich dann die Vorlesung als komplette Videos bei Youtube eingestellt. Die Lösung wurde allg. als sehr günstig beschrieben, da viele Nachteile der Powerpoint-Variante nicht mehr relevant sind.

Nachdem dann auch das Wintersemester nicht in Präsenz möglich war, musste eine Entscheidung über das online-Angebot gefällt werden.

Dabei hatte ich auch an den einfachen Youtube-Videos einige Punkte, die mir nicht gefielen:

- Weiterhin recht schwieriges Navigieren zwischen den einzelnen Folien bzw. Anwahl einer speziellen Folie
- Umständliche Vorbereitung der Videos, wenn auch der Dozent sichtbar sein soll

- und in diesem Fall fehlendes navigieren zwischen diesen Streams

Durch Zufall bin ich dann auf den PaellaPlayer aufmerksam geworden. Dies ist ein Open Source JavaScript Video-Player, der eine unbegrenzte Zahl von Audio- und VideoStreams synchronisiert und diese in unterschiedlichen Formen bereitstellt (u.a. liveStream, ZOOM). Er arbeitet mit allen HTML5 Browsern (Chrome, Firefox, Safari und Edge) sowie unter iOS und Android. Entwickelt wurde der Player im Wesentlichen an der Universitat Politècnica de València und kann für Ausbildungszwecke kostenfrei genutzt werden. [1]

Eine diesbezügliche Nachfrage am Medienzentrums ergab, dass man diesen Player kennt, inn gut findet aber erstmal was Eigenes entwickeln will (?!).

Also wurde ein erster Versuch mit dem originalen Paella-Player in Eigenregie unternommen. Damit wurden alle großen Vorlesungen im ersten Semester (Konstruktionslehre, Informatik) sowie dem fünften Semester (Konstruktiver Entwicklungsprozess) umgesetzt.

\section{Erscheinungsbild und Funktionalität}

Der Student startet wie gewohnt im OPAL. Von dort wird er über einen Link auf eine WEB-Seite umgeleitet, die die Verknüpfungen zu den Vorlesungsvideos enthält. Dies ist erforderlich, da der Paella-Player nicht direkt im OPAL gestartet werden kann.

Die Videos für die Präsentation (z.B. Powerpoint) sowie den Dozenten können lokal oder auch z.B. (wie in unserem Fall) auf Youtube gespeichert sein.

Nach dem Start bietet sich eine Oberfläche mit (zumindest) zwei Videofenstern sowie diversen Funktionsbutton zur Steuerung der Videos an (Abb. 1). 

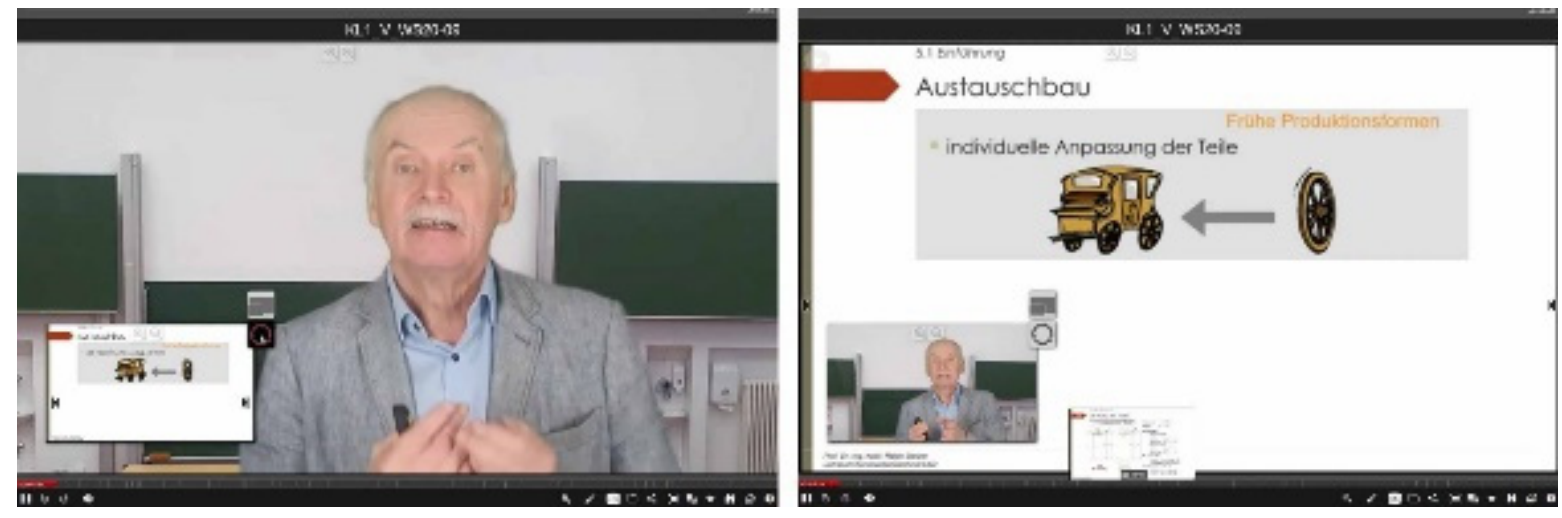

Abb. 1: Varianten des Bildschirmaufbaus bei der Arbeit mit dem Paella-Player

Für die praktische Nutzung stehen jetzt eine Vielzahl unterschiedlicher Funktionen zur Verfügung, wie u.a.:

- Die Vorlesung kann an jeder Stelle beliebig unterbrochen und später fortgesetzt werden.

- Ein Wechsel zwischen der Darstellung der Videos in Größe und Positionierung (Abb. 1 , links bzw. rechts) ist möglich.

- Die einzelnen Videofenster können auch komplett ausgeblendet werden.

- Rücksprung exakt zum Anfang des jeweiligen Slide bzw. zum Anfang des nächsten Slide

- Ansprung eines beliebigen Slide über die Navigationsleiste im unteren Bereich des Viewers. Dort ist über den roten Balken jederzeit zu sehen, wie weit die Vorlesung bereits fortgeschritten ist sowie die Länge jeder einzelnen Szene (Slide).

- Es können auch kleine Bilder jedes Slides im unteren Bildschirmbereich eingeblendet werden, die einen zielgerichtetes Ansprung erleichtern (Abb. 2).

- Umschalten auf Fullscreen

- Festlegen der Videoqualität je nach Geschwindigkeit der WEB-Anbindung

Bereits hier soll auf ein Problem hingewiesen werden, welches sich in der Variante, die wir im Herbst benutzt hatten, ergab.

Da es sich herausgestellt hatte, dass sehr viele Studenten die Vorlesung wirklich simul- tan zu der Zeit anschauen, die im Lehrplan vorgesehen ist, ergibt sich für den StreamingServer eine sehr hohe Last.

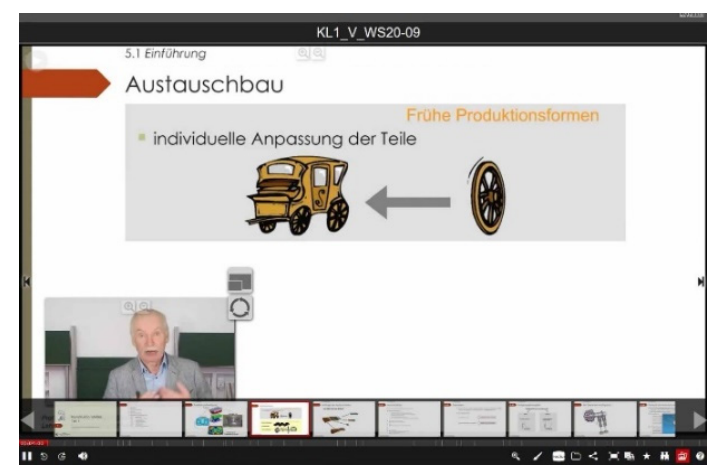

Abb. 2: Navigation zwischen den einzelnen Folien mittels Vorschaubildern

Diese konnte kurzfristig intern nicht gesichert werden. Die Videos wurden deshalb auf die YouTube-Plattform gestellt. Dies hat auch den Vorteil, dass die Videos später einzeln jederzeit weiter zur Verfügung stehen. Allerdings zeigte sich, dass in dem Moment, wo die Vorlesung angehalten wird (um z.B. Inhalte aus dem Präsentationsvideo in das Skript zu übernehmen), YouTube den unteren Teil der Präsentation mit Werbung überlagert. Dieser unschöne Nebeneffekt war kurzfristig leider nicht zu vermeiden, störte aber doch sehr.

Um den Studenten die Arbeit mit diesem bisher ungewohnten Player zu erleichtern wurde ein Einführungsvideo bereitgestellt:

(https://www.youtube.com/watch?v=Axm/2J0pWSk\&t=334s) 


\section{Erstellen der Präsentation}

Erforderlich sind zwei Videos, die beide auf der Serverplattform bereitgestellt werden müssen. Dies sind

- Das Video der Powerpoint-Präsentation sowie

- Das Video des Vortragenden

Darüber hinaus werden benötigt:

- Thumbnail-Bilder für die einzelnen Slides um die direkte Navigation zu erleichtern.

- Ein Strukturfile der die Zuordnung der Thumbnails zu den einzelnen Videoabschnitten enthält und die Navigation in den Videos ermöglicht.

Die Videos sollten im mp4-Format bereitstehen und müssen miteinander synchronisiert sein. Dabei wird das Video des Dozenten mittels einer Kamera (z.B. Handy) aufgenommen. Die Präsentation muss auf dem Bildschirm mitgeschnitten werden. Ich habe dazu das Tool Movavi genutzt. [2]

Da die Videos vom Studenten gestreamt und nicht heruntergeladen werden, müssen diese auf einer so leistungsfähigen Serverplattform abgelegt werden, die den gleichzeitigen $\mathrm{Zu}$ griff der Nutzer (in der Grundlagenvorlesung also von über 500 Personen) gestattet. Aus diesem Grund hatten wir uns, wie bereits erwähnt, für die Ablage bei YouTube entschlossen.

Damit eine Navigation im Viewer zu den einzelnen Slides zielgerichtet möglich ist, wird jeweils ein kleines Vorschaubild benötigt. Diese sollten im jpeg-Format bereitstehen und können bei der Arbeit mit PowerPoint sehr einfach durch "Speichern unter..." erzeugt werden.
Schließlich müssen in einem Strukturfile die Ablagepfade der Videos, die Zeitmarken für den Beginn der einzelnen Abschnitte (meist die Slides) sowie die den Abschnitten jeweils zugeordneten Thumbnails angegeben werden. Dieses File hat das im Internet gebräuchliche json-Format.

Dieses Format ist zwar lesbar, der Aufbau für den Uneingeweihten aber nicht ohne weiteres verständlich und auch relativ aufwendig zu erzeugen.

Aus diesem Grund steht ein Addin für PowerPoint zur Verfügung, welches bei Bedarf in der Menüleiste aktiviert werden kann (Abb. 3).

Dazu muss der Nutzer lediglich ein Flag an der markierten Stelle „Paella-Marken“ setzen. Dadurch erzeugt PowerPoint während der Präsentation das Strukturfile incl. der Zeitmarken für die Folienwechsel synchron zum Video des Dozenten automatisch.

- Unabhängig von der Nutzung des PaellaPlayers stellt übrigens dieses Addin eine Vielzahl weiterer Funktionen zur Unterstützung der Arbeit mit PowerPoint in der Vorlesung bereit, wozu gehören: Umfangreiche Zeichenfunktionen in Vektor- und Pixelgrafik für die Arbeit mit dem Stift bei Ergänzungen in den Folien

- Dynamisches Setzen von Markierungen in Folien zum freien Navigieren während der Vorlesung

- Synchronisierung von Anmerkungen und freien Skizzen zwischen einzelnen Folien

- Einbinden einer Kamera

- Einblendung eines Whiteboards

- Verlinkung eingebetteter Objekte

Darüber kann an anderer Stelle gern informiert werden. Das Video der Präsentation

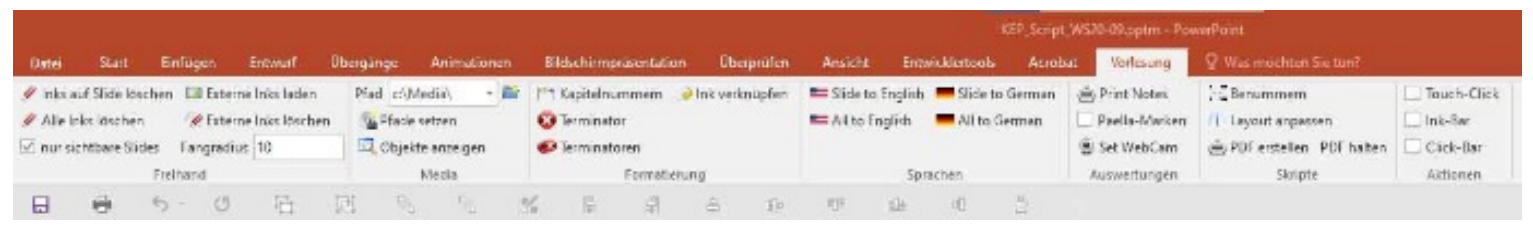

Abb. 3: Oberfläche des Erweiterungs-Addin für Powerpoint 
muss nun noch mit den Zeitmarken und den zugehörigen Thumbnails zu den Folienübergängen synchronisiert werden.

Dazu steht eine weitere Applikation bereit (Abb. 4).

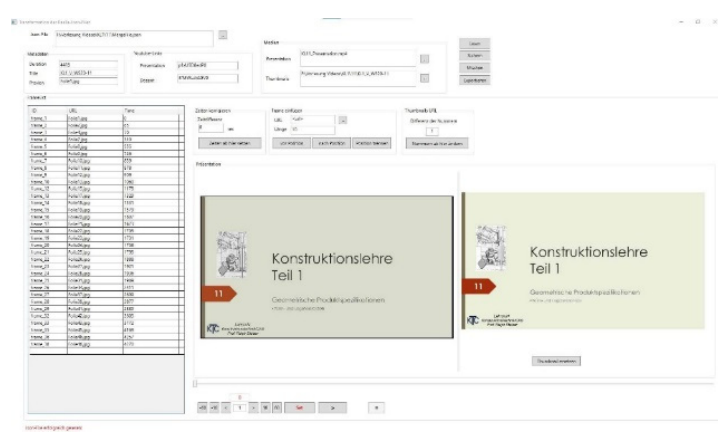

Abb. 4: Bearbeitung der Navigationselemente für die Vorlesung

Dazu wird das Präsentationsvideo geladen sowie der json-Struktur sowie der Pfad der Thumbnails angegeben. Die Übergänge zwischen den Slides können nun manuell mit den angezeigten Thumbnails feinabgestimmt werden. Alternativ können auch weitere Platzhalter eingefügt werden. Dies ist z.B. sinnvoll, wenn in die Präsentation weitere Videos eingebunden werden sollen (z.B. eine später aufgezeichnete Vorführung eines externen Tools, wie z.B. die Arbeit in einem CAD-System.

Schließlich werden alle erzeugten Dateien (zwei Videos, Thumbnails, Strukturfile) auf den Streaming-Server geladen.

\section{Erfahrungen und Weiterführung}

Die Arbeit mit dem Player ist recht komfortabel und hat sich bewährt.

Nachteilig ist der bereits erwähnte Effekt, dass die Streaming-Plattform YouTube teilweise Werbung einblendet.

Wir arbeiten gegenwärtig an einer Lösung, die es ermöglicht, die Videos auf einem anderen Server bereitzustellen.

Das Navigieren innerhalb einer Vorlesung mit diesem Player relativ einfach möglich. Wünschenswert wäre eine Möglichkeit, kontextabhängig Sprungmarken zu setzen, um Inhalte auch über Vorlesungsgrenzen hinweg direkt anzuwählen.
Dies würde den Studenten dann ermöglichen, an Stellen wo auf Inhalte anderer Vorlesungen Bezug genommen wird, im Bedarfsfall diese unmittelbar anzuwählen. Diese Funktion ist grundsätzlich ergänzbar. Voraussetzungen einer Umsetzung werden gegenwärtig untersucht.

\section{Literatur}

[1] Paella Player - the multistream player for lectures https://paellaplayer.upv.es/

[2] Movavi-Screen Recorder https://www.movavi.de 